\title{
Some theorems concerning anti-integral, super-primitive and ultra-primitive elements
}

\author{
Mitsuo Kanemitsu, ${ }^{*}$ Junro Sato** and Ken-IChi Yoshida***
}

Let $R$ be a Noetherian domain and $R[X]$ a polynomial ring. Let $\alpha$ be a non-zero element of an algebraic extension field $L$ of the quotient field $K$ and let $\pi: R[X] \longrightarrow R[\alpha]$ be the $R$-algebra homomorphism sending $X$ to $\alpha$. Let $\varphi_{\alpha}(X)$ be the monic polynomial of $\alpha$ over $K$ with $\operatorname{deg} \varphi_{\alpha}(X)=d$ and write

$$
\varphi_{\alpha}(X)=X^{d}+\eta_{1} X^{d-1}+\cdots+\eta_{d} .
$$

Then $\eta_{i}(1 \leq i \leq d)$ are uniquely determined by $\alpha$. Let $I_{\eta_{i}}=R:{ }_{R} \eta_{i}$ and $I_{[\alpha]}:=\cap_{i=1}^{d} I_{\eta_{i}}$. If $\operatorname{Ker}(\pi)=I_{[\alpha]} \varphi_{\alpha}(X) R[X]$, we say that $\alpha$ is an antiintegral element of degree $d$ over $R$. Put $J_{[\alpha]}=I_{[\alpha]}\left(1, \eta_{1}, \cdots, \eta_{d}\right)$. Then $J_{[\alpha]}=c\left(I_{[\alpha]} \varphi_{\alpha}(X)\right)$, where $c()$ denotes the ideal generated by the coefficients of the polynomials in ( ), that is, the content ideal of ( ). If $J_{[\alpha]} \not \subset p$ for all $p \in \operatorname{Dp}_{1}(R):=\left\{p \in \operatorname{Spec}(R) \mid \operatorname{depth}\left(R_{p}\right)=1\right\}$, then we say that $\alpha$ is a superprimitive element of degree $d$ over $R$. It is known that a super-primitive element over $R$ is anti-integral over $R$ (cf. [3, Theorem 1.12]). It is also known that any algebraic element over a Krull domain $R$ is anti-integral over $R$ (cf. [3, Theorem 1.13]). When $\alpha$ is an element in $K, \varphi_{\alpha}(X)=X-\alpha$. So we have

$$
J_{[\alpha]}=I_{[\alpha]}(1, \alpha)=I_{\alpha}+\alpha I_{\alpha}=I_{\alpha}+I_{\alpha^{-1}} .
$$

where $I_{\alpha}:=R:{ }_{R} \alpha$. In this case, $\alpha \in K$ is anti-integral over $R$ if and only if $R[\alpha] \cap R\left[\alpha^{-1}\right]=R$ (cf. [3]).

N. Onoda, T. Sugatani and K. Yoshida, for any $a \in R$, asked whether equality $R[\alpha] \cap R[1 / \alpha]=R[\alpha] \cap R[1 /(\alpha-a)]$ is always true and gave an affirmative answer in $d \leq 4$. Hence, in this case, the set $\{R[\alpha] \cap R[1 /(\alpha-a)] \mid a \in R\}$ is a one point set.

In this paper, we considered the set $\left\{B_{a} \mid a \in R^{*}\right\}$, where $R^{*}=R-\{0\}$ and $B_{a}=R[a \alpha] \cap R[1 / a \alpha]$. Under some conditions, we shall show that $\left\{B_{a}\right\}$ $\left.a \in R^{*}\right\} \cong R^{*} / \mathrm{U}(R)$, where $\mathrm{U}(R)$ denotes the unit group of $R$.

Received September 14, 1998.

1991 Mathematics Subject Classification. 13B02.

* Department of Mathematics, Aichi University of Education, Igaya-cho, Kariya-shi 448-8542, Japan.

** Department of Management information, Southern Osaka University, Hirao, MinamiKawachi 1060-1, Osaka 587-8555, Japan.

*** Department of Applied Mathematics, Okayama University of Science, Ridai-cho, Okayama 700-0005, Japan. 
Throughout this paper, we use the following notation unless otherwise specified:

Let $R$ be an Noetherian domain with quotient field $K$, Let $\bar{R}$ denote the integral closure of $R$ in $K$. Then $\bar{R}$ is a Krull domain (cf. [2]). Let $c(\bar{R} / R$ ) denote the conductor ideal, i.e. $\{a \in R \mid a \bar{R} \subset R\}$. Let $L$ be an algebraic extension field of $K$ and let $\alpha$ be an element in $L$ which is degree $d$ over $K$. Let $\varphi_{\alpha}(X):=X^{d}+\eta_{1} X^{d-1}+\cdots+\eta_{d}$ denote the minimal polynomial of $\alpha$ over $K$ (that is $\eta_{i} \in K$ ). Let $I$ be an ideal of $R$. When $I \neq R$, then we define $\operatorname{grade}(I):=$ the length of a maximal $R$-sequence in $I$. When $I=R$, then we define grade $(I):=\infty$ by convension (cf. [2]).

An element $\alpha \in L$ is called to be ultra-primitive over $R$ if $\alpha$ satisfies grade $\left(I_{[\alpha]}+c(\bar{R} / R)\right)>1$. Note that $c(\bar{R} / R)$ may be $(0)$. In this case, if $\alpha \in K$ is ultra-primitive over $R$, then the condition grade $\left(I_{[\alpha]}+c(\bar{R} / R)\right)>1$ implies that $I_{[\alpha]}=R$ because grade $\left(I_{[\alpha]}\right)=1$ or $I_{[\alpha]}=R$, and hence that $\alpha \in R$. It is known that an ultra-primitive element is a super-primitive element and hence an anti-integral element (cf. [4]).

Our general reference for unexplained technical terms is [2].

We start with the following lemma.

Lemma 1. Let $a, b$ be elements of $R$. Assume that $a$ and $b$ are non-zero divisors on $R / I_{[\alpha]}$. Then $I_{[\alpha]}=I_{[a \alpha]}=I_{[b \alpha]}$ hold. If, in addition, $\alpha$ is superprimitive, then $a I_{[\alpha]}=b I_{[\alpha]}$ implies that $a R=b R$.

Proof. First we shall show that $I_{[\alpha]}=I_{[a \alpha]}$. Since $\varphi_{a \alpha}(X):=X^{d}+$ $a \eta_{1} X^{d-1}+a^{2} \eta_{2} X^{d-2}+\cdots+a^{d} \eta_{d}$ is the minimal polynomial of $a \alpha$ of degree $d$ over $K$, we have $I_{[\alpha]} \subset \cap_{i=1}^{d} I_{a^{i} \eta_{i}}=I_{[a \alpha]}$ and $I_{[a \alpha]}=\cap_{i=1}^{d} I_{a^{i} \eta_{i}}=\cap_{i=1}^{d}\left(I_{\eta_{i}}: a^{i}\right) \subset$ $\cap_{i=1}^{d}\left(I_{\eta_{i}}: a^{d}\right)=\left(\cap_{i=1}^{d} I_{\eta_{i}}\right): a^{d}=I_{[\alpha]}: a^{d}$.

Since $a$ is a non-zero divisor on $R / I_{[\alpha]}$, it follows $I_{[\alpha]}=I_{[a \alpha]}$.

Next we assume that $\alpha$ is super-primitive. We have only to show that $b / a \in R$. In order to prove that $b / a \in R$, it sufficies that $b / a \in R_{p}$ for every $p \in \mathrm{D} p_{1}(R)$. If $p \not \supset I_{[\alpha]}$, then $\left(I_{[\alpha]}\right)_{p}=R_{p}$, so $a R_{p}=a\left(I_{[\alpha]}\right)_{p}=b\left(I_{[\alpha]}\right)_{p}=b R_{p}$. Hence $b / a \in R_{p}$. If $p \supset I_{[\alpha]}$, since $\alpha$ is super-primitive, then $I_{[\alpha]} R_{p}=x R_{p}$ for some $x \in I_{[\alpha]}$ by [3, Theorem 2.11]. Thus $a I_{[\alpha]} R_{p}=a x R_{p}=b x R_{p}=b I_{[\alpha]} R_{p}$, and hence $a R_{p}=b R_{p}$. Therefore $b / a \in R_{p}$. Thus we have $b / a \in \cap_{p \in \mathrm{Dp}_{1}(R)} R_{p}=R$ by [6], as desired.

Lemma 2. Let a be an element of $R$. Assume that $a$ is a non-zero divisor on $R / I_{[\alpha]}$. Let $\alpha$ be a super-primitive element of degree $d$ over $R$. Then a $\alpha$ is a super-primitive element over $R$.

Proof. We have

$$
J_{[a \alpha]}=I_{[a \alpha]}\left(1, a \eta_{1}, \cdots, a^{d} \eta_{d}\right)=I_{[\alpha]}\left(1, a \eta_{1}, \cdots, a^{d} \eta_{d}\right)
$$

by Lemma 1. Suppose that $p \supset J_{[a \alpha]}$ for some $p \in \operatorname{Dp}_{1}(R)$. Then $I_{[\alpha]} \subset$ $I_{[\alpha]}\left(1, a \eta_{1}, \cdots, a^{d} \eta_{d}\right)$. Since $\alpha$ is super-primitive over $R, p$ is a prime divisor 
of $I_{[\alpha]}$. Hence $a \notin p$ by assumption. Since $a^{i} \eta_{i} I_{[\alpha]} \subset p(1 \leq i \leq d)$, it holds $\eta_{i} I_{[\alpha]} \subset p$. Therefore we obtain $J_{[\alpha]}=I_{[\alpha]}\left(1, \eta_{1}, \cdots, \eta_{d}\right) \subset p$. This contradicts that $\alpha$ is super-primitive over $R$. Hence we see that $p \not \supset J_{[a \alpha]}$ for any $p \in \mathrm{Dp}_{1}(R)$. This yields that $a \alpha$ is super-primitive over $R$.

Let $\alpha$ be an anti-integral element of degree $d$ over $R$. Then it is well-known that

$R[\alpha] \cap R\left[\alpha^{-1}\right]=R \oplus I_{[\alpha]} \zeta_{1} \oplus \cdots \oplus I_{[\alpha]} \zeta_{d-1}$, where $\zeta_{i}=\alpha^{i}+\eta_{1} \alpha^{i-1}+\cdots+$ $\eta_{i}(1 \leq i \leq d-1)$, by [1, Theorem 1$]$.

Theorem 3. Let $R$ be a Noetherian domain with quotient field $K$. Let $\alpha$ be a super-primitive element of degree $d(\geq 2)$ over $R$ and let $a, b \in R$. Assume that $a$ and $b$ are non-zero divisors on $R / I_{[\alpha]}$. Let $B_{a}=R[a \alpha] \cap R[1 /(a \alpha)]$ and $B_{b}=R[b \alpha] \cap R[1 /(b \alpha)]$. Then the following statements are equivalent.

(1) $B_{a}=B_{b}$.

(2) $a=u b$ for some $u \in \mathrm{U}(R)$.

Proof. $(1) \Longrightarrow(2): I_{[\alpha]}=I_{[a \alpha]}=I_{[b \alpha]}$ by Lemma 1. Since $\alpha$ is superprimitive and $a, b$ are non-zero divisors on $R / I_{[\alpha]}$, it follows that $a \alpha, b \alpha$ are super-primitive over $R$ by Lemma 2 , and hence anti-integral over $R$. Therefore we have the direct sum decompositions of $B_{a}, B_{b}$ as follows:

$$
\begin{aligned}
B_{a} & =R \oplus I_{[a \alpha]} a \zeta_{1} \oplus \cdots \oplus I_{[a \alpha]} a^{d-1} \zeta_{d-1} \\
& =R \oplus I_{[\alpha]} a \zeta_{1} \oplus \cdots \oplus I_{[\alpha]} a^{d-1} \zeta_{d-1} \\
B_{b} & =R \oplus I_{[b \alpha]} b \zeta_{1} \oplus \cdots \oplus I_{[b \alpha]} b^{d-1} \zeta_{d-1} \\
& =R \oplus I_{[\alpha]} b \zeta_{1} \oplus \cdots \oplus I_{[\alpha]} b^{d-1} \zeta_{d-1} .
\end{aligned}
$$

Note that $\zeta_{i}=\alpha^{i}+\eta_{1} \alpha^{i-1}+\cdots+\eta_{i}(1 \leq i \leq d-1)$ by [1]. Since $B_{a}=B_{b}$ and $d \geq 2$, we conclude $I_{[\alpha]} b \zeta_{1}=I_{[\alpha]} a \zeta_{1}$, and hence $a I_{[\alpha]}=b I_{[\alpha]}$. Thercfore, by Lemma 1 , it holds $a / b \in R$, and thus $a=u b$ for some $u \in \mathrm{U}(R)$, as desired.

$(2) \Longrightarrow(1)$ : Since $a=u b$ for some $u \in \mathrm{U}(R)$, we conclude $I_{[\alpha]} a^{i} \zeta_{i}=I_{[\alpha]} b^{i} \zeta_{i}$ for all $i(1 \leq i \leq d-1)$. Hence $B_{a}=B_{b}$ by $\left(^{*}\right)$.

Corollary 1. Let $S=R-\cup p_{i}\left(p_{i} \in \operatorname{Ass}\left(R / I_{[\alpha]}\right)\right)$. We define the mapp$\operatorname{ping} \Phi: S \longrightarrow\left\{B_{a} \mid a \in S\right\}$ sending $a \in S$ to $B_{a}$. Then $\operatorname{Ker}(\Phi)=S / \mathrm{U}(R)$ by Theorem 3 , and hence we have the bijection $\bar{\Phi}: S / \mathrm{U}(R) \cong\left\{B_{a} \mid a \in S\right\}$.

Lemma 4. Let $\alpha$ be an ultra-primitive element over $R$. Then, for any $a \in$ $R, a \alpha$ is ultra-primitive, therefore a $\alpha$ is anti-integral over $R$.

Proof. Since $\alpha$ is ultra-primitive, then $\alpha$ is anti-integral. Hence $\alpha$ is antiintegral over $R_{p}$ for any $p \in \mathrm{Dp}_{1}(R)$ by [5]. If $p \not \supset c(\bar{R} / R)$, then $R_{p}$ is normal, and hence $a \alpha$ is anti-integral by [3]. If $p \not \supset I_{[\alpha]}$, then $\alpha$ is integral over $R_{p}$ ([3, Corollary 2.3]), and thus $a \alpha$ is integral over $R_{p}$. Note that $a \alpha$ has monic relation of degree $d$. Therefore $a \alpha$ is anti-integral over $R_{p}$. We are done.

Next we study the set $\{R[1 / a \alpha] \mid a \in R, a \alpha$ is anti-integral over $R\}$. Under some conditions, we shall show that the above set correspondent to $R^{*} / \mathrm{U}(R)$ bijectively. 
Theorem 5. Let $R$ be a Noetherian domain with quotient field $K$. Let $\alpha$ be a ultra-primitive element of degree $d(\geq 3)$ over $R$. Let $a, b \in R^{*}$ and let $\bar{R}$. be the integral closure of $R$. Suppose that $\bar{R}$ is a finite $R$-module. Let $B_{a}=$ $R[a \alpha] \cap R[1 /(a \alpha)]$ and $B_{b}=R[b \alpha] \cap R[1 /(b \alpha)]$. Then the following statements are equivalent.

(1) $B_{a}=B_{b}$.

(2) $a=u b$ for some $u \in \mathrm{U}(R)$.

Proof. $(2) \Longrightarrow(1)$ : Since $\alpha$ is ultra-primitive over $R, a \alpha$ and $b \alpha$ are antiintegral over $R$ by Lemma 4 . The rest of this implication is proved in the same way as the proof of Theorem $3((2) \Longrightarrow(1))$.

$(1) \Longrightarrow(2)$ : Since $a \alpha$ and $b \alpha$ are anti-integral by Lemma 4 , we have the decompositions

$$
\begin{aligned}
& B_{a}=R \oplus I_{[a \alpha]} a \zeta_{1} \oplus I_{[a \alpha]} a^{2} \zeta_{2} \oplus \cdots \oplus I_{[a \alpha]} a^{d-1} \zeta_{d-1} \\
& B_{b}=R \oplus I_{[b \alpha]} b \zeta_{1} \oplus I_{[b \alpha]} b^{2} \zeta_{2} \oplus \cdots \oplus I_{[b \alpha]} b^{d-1} \zeta_{d-1}
\end{aligned}
$$

Since $d \geq 3$, we have $a I_{[a \alpha]}=b I_{[b \alpha]}$ and $a^{2} I_{[a \alpha]}=b^{2} I_{[b \alpha]}$. It follows that $b^{2} I_{[b \alpha]}=a^{2} I_{[a \alpha]}=a b I_{[b \alpha]}$, and hence $b I_{[b \alpha]}=a I_{[b \alpha]}$. Therefore $a=u b$ for some $u \in \mathrm{U}(R)$ by Lemma 1 , as desired.

COROllary 2. By the above theorem, if $\alpha$ is ultra-primitive, then we have the bijection $\Phi: R^{*} / \mathrm{U}(R) \cong\left\{B_{a} \mid a \in R^{*}\right\}$ defined by $\Phi(\operatorname{amodU}(R))=B_{a}$ for any $a \in R^{*}$.

\section{References}

[1] M.Kanemitsu and K.Yoshida, Some properties of extensions $R[\alpha] \cap R\left[\alpha^{-1}\right]$ over Noetherian domains $R$, Communications in Algebra 23 (1995), 4501-4507.

[2] H.Matsumura, Commutative Algebra, Benjamin, New York, 1970.

[3] S.Oda, J.Sato and K.Yoshida, High degree anti-integral extensions of Noetherian domains, Osaka J. Math. 30 (1993), 119-135.

[4] S.Oda and K.Yoshida, A linear generator of ultra-primitive extensions $R[\alpha]$, Far East J. Math. Sci. 6 (1998), 65-74.

[5] J.Sato, S.Oda and K.Yoshida, A characterization of anti-integral extensions, Math. J. Okayama Univ. 37 (1995), 55-58.

[6] K.Yoshida, On birational-integral extensions of rings and prime ideals of depth one, Japan J. Math. 8 (1982), 49-70. 